\title{
Análisis desde una perspectiva tributaria de la implementación de negocios de crowdfunding o financiamiento participativo basados en el modelo de préstamo
}

\author{
Wilfredo Mego Chavez \\ Abogado por la Universidad Nacional Mayor de San Marcos. \\ Maestrando de la Maestría de Economía con Mención en Finanzas en la
Universidad Nacional Mayor de San Marcos.
}

SUMARIO:

I. Introducción.

II. Aproximación conceptual al negocio de crowdfunding.

III. Experiencia en el Perú en la estructuración de negocios de crowdfunding bajo el modelo de préstamo.

IV. Principales aspectos tributarios a tener en consideración.

1. Respecto a la relación jurídica realizada entre el promotor y los inversionistas.

2. Respecto a las relaciones jurídicas realizadas entre el administrador de la plataforma, el promotor y los inversionistas.

3. Respecto a la obligación de emitir comprobantes de pago.

4. Consideraciones adicionales.

v. Conclusiones. 


\section{RESUMEN:}

Para la implementación de nuevos negocios y proyectos mediante el uso de las Tecnologías de la Información y las Comunicaciones — TIC —, resulta necesario contar con un soporte tributario adecuado y permanente, puesto que la inobservancia de los aspectos fiscales de una operación podría determinar sobrecostos para los emprendedores al generarse cargas impositivas muy elevadas o incluso la pérdida de beneficios.

En ese contexto, el presente artículo tiene como objetivo analizar desde una perspectiva tributaria la implementación de negocios de crowdfunding o financiamiento participativo bajo el modelo de préstamo; toda vez que constituye un mecanismo de financiamiento emergente, en el cual los fundadores de nuevos negocios y proyectos han encontrado una alternativa atractiva frente a los modelos convencionales - p.e. préstamos bancarios-.

Palabras clave: Financiamiento participativo, crowdfunding, impuestos, mercado financiero, fintech, servicios financieros, Impuesto a la Renta, Impuesto General a las Ventas.

\footnotetext{
ABSTRACT:

For the implementation of new businesses and projects with Information and Communication Technologies — ICT_, it is necessary to have an adequate and permanent tax support, since the non-compliance of the tax aspects of an operation could result in cost overruns for entrepreneurs by generating very high tax burdens or even the loss of benefits.

In this context, the purpose of this article is to analyze from a tax perspective the implementation of crowdfunding or participatory financing businesses under the loan model; since it constitutes an emerging financing mechanism, in which the founders of new businesses and projects have found an attractive alternative to conventional models - e.g. bank loans-.

Keywords: Participative financing, crowdfunding, taxes, financial market, fintech, financial services, Income Tax, General Sales Tax.
}

\section{INTRODUCCIÓN}

El acercamiento al mundo digital mediante la utilización de las Tecnologías de la Información y las Comunicaciones - TIC en la realización de los negocios se ha acelerado en los últimos años de tal manera que actualmente es usual utilizar un medio digital para contratar un servicio o adquirir algún bien. Esta situación no es ajena al mercado financiero. Éste se ha visto ampliamente influenciado por las innovaciones tecnológicas al momento de implementar diferentes modelos de negocios que son recogidos bajo el concepto de fintech. ${ }^{1}$

Así pues, las fintech se manifiestan en el mercado financiero de dos formas: por un lado, las
Empresas del Sistema Financiero² están integrando las TIC en la ejecución de sus actividades — por ejemplo, implementando sistemas de pago digitales, aplicativos móviles, Big Data, entre otros-; y por otro lado, nuevas empresas han visto la posibilidad de competir mediante su uso con las entidades financieras tradicionales, ya que las innovaciones tecnológicas están permitiendo reducir las limitaciones que restringían su acceso a competir en el mercado financiero.

En este segundo grupo se presentan diferentes tipos de iniciativas - por ejemplo, soluciones de pago, casas de cambio online, entre otrossiendo una de ellas el crowdfunding o financiamiento participativo - por su traducción al es-

1. Giorgio Beretta, "VAT on Financial and Insurance Services at the Dawn of the Fourth Industrial Revolution", International VAT Monitor 29 n4 (2018): 143.

2. El sistema financiero es definido por la Ley 26702 - Ley General del Sistema Financiero y del Sistema de Seguros y Orgánica de la Superintendencia de Banca y Seguros como: "El conjunto de empresas, que debidamente autorizadas operan en la intermediación financiera; se incluye a las subsidiarias que requieran de autorización de la SBS para constituirse". 
pañol ${ }^{3}-$, mediante el cual una persona natural o jurídica - usualmente empresas que recién están iniciando operaciones- busca financiarse por un gran grupo de inversores. ${ }^{4}$ Este modelo de negocio ha crecido de forma exponencial debido a que permite a los usuarios acceder a servicios de financiamiento alternativo de forma ágil y sencilla, puesto que se realiza a través de una plataforma digital. ${ }^{5}$

No obstante, en tanto se trata de un modelo de negocio relativamente nuevo, éste trae consigo una serie de riesgos para los partícipes, puesto que no cuentan aún con un marco normativo consolidado que regule todos los elementos propios de este tipo de fintech, siendo uno de ellos el aspecto tributario.

Tener claro el marco normativo tributario al mo- mento de estructurar un negocio resulta necesario $^{6}$ para cualquiera que desee desarrollar un nuevo emprendimiento; por dicha razón, consideramos importante abordar desde esta perspectiva la implementación del negocio de crowdfunding, analizando el impacto que eventualmente podría tener en cada uno de los partícipes según las relaciones jurídicas que se lleven a cabo.

Para efectos de determinar dichas relaciones jurídicas, se partirá de un análisis conceptual del alcance del negocio de crowdfunding, así como de los modelos bajo los cuales se han estado estructurando en el mercado peruano.

\section{APROXIMACIÓN CONCEPTUAL AL NEGO- CIO DE CROWDFUNDING}

El crowdfunding ${ }^{7}$, de manera general, es defi-

3. Para efectos del presente trabajo adoptaremos el término original en inglés.

4. Cathalene Bowler y Dennis Schmidt lo definen en ese sentido: "Crowdfunding is the process of asking a large number of people to contribute money to fund a particular activity. Typically, fundraisers do this via the Internet through a third-party intermediary called a "platform." Crowdfunding campaigns vary widely and include endeavors such as donating to a charity, covering personal expenses, funding a creative project, or raising debt or equity capital for a startup business - among a host of other causes-". Cathalene Bowler y Dennis Schmidt, "Crowdfunding: Tax Issues and Strategies", Practical Tax Strategies 99 n 3, (2017): 20.

5. De acuerdo con el Decreto Supremo N²55-2019-EF —Aprueban la Política Nacional de Inclusión Financiera y modifican el Decreto Supremo No 029-144-EF- aunque incipiente, el desarrollo de las fintech en el Perú reviste gran potencial. Así, además de otros servicios financieros, las fintech ofrecen financiamiento sea con recursos propios, actuando como intermediario - $\mathrm{P} 2 \mathrm{P}$ - , o a través de financiamiento participativo financiero - crowdfunding - , con el cual las personas o empresas pueden acceder a fondos provistos por una pluralidad de pequeños inversionistas.

6. Los cambios en los modelos de negocio - como es el caso de las fintech - como resultado de la digitalización de la economía mediante la utilización de las TIC representan un desafío para las políticas tributarias y fiscales de los Estados, toda vez que los sistemas tributarios se adecúan con lentitud y muchas veces de forma inadecuada generando espacios que permiten la erosión de ingresos fiscales. Así por ejemplo la Comisión Económica para América Latina y el Caribe - CEPAL señala que "la digitalización ha permitido que algunas empresas participen activamente en determinados sectores económicos de varios países, sin tener necesariamente una presencia física significativa en ellos. Por el lado del impuesto al valor agregado -IVA-, surge la dificultad para gravar las operaciones en el lugar de consumo, sobre todo en el caso de servicios digitales, toda vez que el vendedor reside en otra jurisdicción. En el caso del impuesto a la renta, al no existir un establecimiento permanente en el país en que se realizan las operaciones, las rentas de la empresa solo pueden gravarse en el país en que reside. En este sentido, la digitalización de la economía exacerba el desvío de las utilidades hacia jurisdicciones de baja tributación, por la facilidad para escoger cualquier jurisdicción como país de residencia y centro de operaciones". Comisión Económica para América Latina y el Caribe, Panorama Fiscal de América Latina y el Caribe 2019: políticas tributarias para la movilización de recursos en el marco de la Agenda 2030 para el Desarrollo Sostenible (Santiago: CEPAL, 2019): 49.

7. Según el Banco Mundial, el financiamiento participativo se originó como consecuencia de la crisis del 2008, como un mecanismo para la canalización de donaciones o aportes voluntarios. Crowdfunding's Potential for Developing World, (Washington, D.C.: World Bank Group, 2013): 14. 
nido como aquel modelo de financiamiento que se lleva a cabo mediante una plataforma digital donde pequeñas cantidades de dinero provenientes de un gran número de personas -naturales o jurídicas - financian un proyecto, negocio u otra necesidad ya sea con fines altruistas o a cambio de obtener cierta renta-

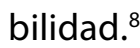

Para su implementación ${ }^{9}$ se requiere la intervención de tres sujetos que se interrelacionan constantemente en el desarrollo del negocio: la plataforma - que usualmente es administrada por una persona jurídica - viene a ser el soporte digital a través del cual se contactan el promotor, como agente deficitario que busca financiamiento, y el inversionista que actuará como agente superavitario.

Así pues, la plataforma digital actúa como un medio para conectar la oferta con la demanda de fondos, permitiendo realizar a los inversionistas aportes dinerarios a favor de los promotores $y$, de ser el caso, transferir la rentabilidad obtenida -intereses o dividendos - una vez concluido el negocio o la operación a favor de aquellos. Es así como la plataforma podrá ac- tuar únicamente como intermediaria, o bien desarrollar actividades adicionales como recibir el dinero de los inversionistas y transferirlo al promotor o inclusive realizar acciones de cobranza cuando correspondan. ${ }^{10}$

La única característica estándar del crowdfunding será la participación de dichos sujetos, siendo que los demás elementos - por ejemplo, tipo de rentabilidad, tipo de negocio o proyecto, etc.- dependerán de sus necesidades y objetivos, no existiendo un modelo bajo el cual puedan regirse o establecerse las reglas de su operatividad.

Una forma de clasificar las diferentes maneras bajo las cuales se han venido estructurando este tipo de negocio es en base al tipo de retorno esperado por parte de los inversionistas, dividiéndose en no financieros y financieros. En el primer grupo encontraremos los denominados de donación y de recompensa, y en el segundo grupo estarán el de capital y préstamo - esta última categoría se puede presentar de diferentes maneras, como por ejemplo mediante operaciones de factoring - .." Esta clasificación puede ser esquematizada de la siguiente manera:

8. El Inter-American Development Bank define al crowdfunding de la siguiente manera: "It consists of enterprises, namely platforms or originators, using electronic means to match supply from investors or donors with the demand for funds, represented by promoters (individual or enterprises) who have specific projects to be funded". Diego Herrera, Alternative Finance (Crowdfunding) Regulation in Latin America and the Caribbean, (Inter-American Development Bank, 2016): 3.

9. Como señalan Rafael J. Sáenz Gómez y Manuel Lucas Durán: “Las operaciones de financiación colectiva obedecen a esquemas que conllevan una cierta complejidad, de manera que para proceder a su análisis es conveniente desentrañar las relaciones jurídicas que se presentan en tales supuestos. Así pues, puede indicarse que habitualmente existirán al menos 3 sujetos implicados: quien realiza la aportación, quien la recibe y quien pone en contacto a uno y a otro sujeto (plataforma mediadora)". Rafael Sáenz Gómez y Manuel Lucas Durán, "Implicancias tributarias del Crowdfunding o financiación colectiva", Quincena Fiscal 9 (2015): 24.

10. Madeleine Merkx, "The VAT Consequences of Crowdfunding", International VAT Monitor 27 n० 1 (2016): 12.

11. Dicha clasificación es la que se recoge con mayor frecuencia a nivel doctrinario: "Depending on the return that can be obtained by the investor, one can widely classify crowdfunding using the following forms: donation, loan based, reward based and equity based". Gramage, F. y Beltran, J., "Spain - Tax Implications of Various Crowdfunding Models: A General Overview from a Spanish Perspective", Finance and Capital Markets 16 n 1, (2014): 3.

12. La intención de incorporar un marco normativo aplicable a las operaciones de financiamiento participativo viene desde el 2017, mediante los Proyectos de Ley No 3080/2017-CR del 27 de junio de 2018, 3403/2018-CR, del 18 de setiembre de 2018, 4324/2018-CR, del 13 de mayo de 2019, y 4421/2019-CR, del 05 junio de 2019. 


\begin{tabular}{|c|c|c|c|}
\hline Fines & $\begin{array}{l}\text { Modalidad de } \\
\text { Crowdfunding }\end{array}$ & $\begin{array}{l}\text { Tipo de proyecto } \\
\text { y/o negocio }\end{array}$ & $\begin{array}{l}\text { Retribución para } \\
\text { los inversionistas }\end{array}$ \\
\hline \multirow{2}{*}{ No financieros } & $\begin{array}{l}\text { De donación } \\
\text { —donation-based } \\
\text { crowdfunding- }\end{array}$ & $\begin{array}{l}\text { - Proyectos sociales } \\
\text { y/o humanitarios. } \\
\text { - Sin fines lucrativos, } \\
\text { no hay un beneficio } \\
\text { para los inversionistas. }\end{array}$ & No \\
\hline & $\begin{array}{l}\text { De recompensa } \\
\text { —reward-based } \\
\text { crowdfunding- }\end{array}$ & $\begin{array}{l}\text { - Proyectos culturales } \\
\text { o creativos. } \\
\text { - Los inversionistas obtienen } \\
\text { una recompensa que está } \\
\text { materializada en un bien } \\
\text { o servicio. }\end{array}$ & No \\
\hline \multirow{2}{*}{ Financieros } & $\begin{array}{l}\text { De capital } \\
\text { — equity-based } \\
\text { crowdfunding- }\end{array}$ & $\begin{array}{l}\text { - Proyectos de índole } \\
\text { empresarial. } \\
\text { El beneficio de los } \\
\text { inversionistas puede } \\
\text { constituir la participación } \\
\text { en los rendimientos } \\
\text { de un negocio o en el } \\
\text { capital social de la empresa. }\end{array}$ & Sí \\
\hline & $\begin{array}{l}\text { De préstamo } \\
\text {-lending-based } \\
\text { crowdfunding- }\end{array}$ & $\begin{array}{l}\text { - Diferentes tipos } \\
\text { de proyectos. } \\
\text { - Bajo esta modalidad } \\
\text { los proyectos ofrecen } \\
\text { como beneficio el pago } \\
\text { de un interés por el uso } \\
\text { del dinero en el tiempo; } \\
\text { en base a ello bajo esta } \\
\text { modalidad la relación } \\
\text { jurídica sería la equivalente } \\
\text { a un préstamo o mutuo. }\end{array}$ & Sí \\
\hline
\end{tabular}

Para efectos del presente trabajo, nos centraremos en el análisis fiscal del crowdfunding bajo el modelo de préstamo, puesto que como se evidenciará más adelante, es el modelo utilizado por las plataformas digitales existentes en el mercado peruano, además que viene a ser una de las modalidades recogidas por el marco regulatorio vigente.

En efecto, desde el 23 de enero de 2020 se encuentra vigente el Decreto de Urgencia 013-
2020 que promueve el financiamiento de la MIPYME - micro, pequeña y mediana empresa-, emprendimientos y Startups; y como parte de sus objetivos busca promover e impulsar la actividad de financiamiento participativo financiero como instrumento que permita un mayor acceso al financiamiento, estableciendo un marco jurídico para regular y supervisar su actividad, así como a las sociedades autorizadas para administrar las plataformas a través de las cuales se realizarían dichas actividades. ${ }^{12}$ 
Para dichos efectos, se define como tal a la actividad en la que a través de una plataforma pone en contacto a personas naturales domiciliadas en el país o personas jurídicas constituidas en el país, que solicitan financiamiento a nombre propio, denominados receptores, con una pluralidad de personas naturales, jurídicas o entes colectivos, denominados inversionistas, que buscan obtener un retorno financiero. Se excluye expresamente de su alcance a aquellos donde los inversionistas - $u$ ofertantes - no persigan un retorno financiero y en los cuales no se pueda evidenciar la existencia del aspecto colectivo de la operación.

En cuanto a las modalidades bajo las cuales se puede llevar a cabo, se ha recogido de forma expresa la realizada mediante valores representativos de capital y/o deuda y la de préstamo; no obstante, se deja abierta la posibilidad que la Superintendencia de Mercado de Valores peruana - SMV regule otras modalidades mediante normas de carácter general.

Respecto a los partícipes, se ha dispuesto que la administración de la plataforma únicamente puede ser llevada a cabo por sociedades anónimas constituidas en el Perú ${ }^{13}$ que tengan como objeto social la administración de dichas plataformas y estén debidamente autorizadas por la SMV; además de las Empresas del Sistema Financiero comprendidas en el artículo 16 de la Ley 26702 - Ley General del Sistema Financiero y del Sistema de Seguros y Orgánica de la Superintendencia de Banca y Seguros. ${ }^{14}$

En relación con los promotores, se tiene que estos serán personas naturales o jurídicas que deberán calificar como sujetos domiciliados en el Perú según la definición de financiamiento participativo financiero recogida, la cual únicamente hace alusión expresa a personas naturales domiciliadas en el país o personas jurídicas constituidas en el país. Por su parte, tratándose de los inversionistas se tiene que estos podrán ser personas naturales, personas jurídicas y entes colectivos - fondos de inversión, fondos mutuos de inversión en valores, así como fideicomisos bancarios y de titulización-, y no se excluye a los sujetos no domiciliados.

\section{EXPERIENCIA EN EL PERÚ EN LA ESTRUC- TURACIÓN DE NEGOCIOS DE CROWDFUN- DING BAJO EL MODELO DE PRÉSTAMO}

Una de las particularidades de los negocios de crowdfunding es que no existe una modalidad típica. Por lo tanto, pueden ser estructurados de diferente manera y bajo diferentes tipos de relaciones jurídicas entre el promotor, los inversionistas y el administrador de la plataforma. Dicha estructura deberá ser claramente identificada a efectos de determinar las implicancias fiscales.

Si bien las ideas expuestas en el punto II nos dan un marco general de cómo se estructura este tipo de negocios, consideramos oportuno revisar la experiencia del mercado peruano a fin de tener un panorama más amplio.

Para dichos efectos, hemos revisado algunas plataformas que actualmente operan en el Perú, tales como: Afluenta ${ }^{15}$, Facturedo ${ }^{16}$, Di-

13. Sin perjuicio de ello, mediante su Decimocuarta Disposición Complementaria Final, ha previsto que tratándose de la administración de plataformas por empresas no constituidas en el Perú, el requisito de que la administración de las plataformas se lleve a cabo por sociedades anónimas constituidas en el país es aplicable en tanto no contravenga los tratados internacionales de los cuales el Perú es parte y siempre que la sociedad administradora o su equivalente se encuentre constituida en el país con el cual el Perú haya celebrado dichos tratados. Agrega que la sociedad administradora o su equivalente debe encontrarse bajo el ámbito de un organismo supervisor de los servicios señalados en el numeral 24.1 de su artículo 24.

14. En el citado artículo se regula a las empresas de operaciones múltiples, empresas especializadas, bancos de inversión y empresas de seguros.

15. "Afluenta | Finanzas colaborativas “, Afluenta, acceso el 21 de marzo de 2021, https://www.afluenta.pe/

16. "Facturedo - Factoring online en Perú - Descuento de facturas online Perú", Facturedo, acceso el 21 de marzo de 2021, https://facturedo.pe/ 
fondy ${ }^{17}$, AirOrHouse ${ }^{18}$, Prestamype ${ }^{19}$, Agroinvesting ${ }^{20}$, Finsmart ${ }^{21}$ y Creafunding ${ }^{22}$, las cuales son manejadas por empresas domiciliadas en el Perú y buscan permitir interactuar a los inversionistas y promotores con el objetivo de realizar operaciones de préstamos, y en algunas operaciones de factoring.

Respecto a la modalidad bajo la cual se han procedido a estructurar, se tiene que en general han optado por suscribir contratos de mandato - esencialmente con representaciónentre los inversionistas y el administrador de la plataforma, en virtud del cual este último recibe instrucciones de aquellos a efectos realizar préstamos. De forma similar, en algunos casos se ha evidenciado que también se procede a suscribir contratos de mandato con el operador.

Por su parte, a efectos de garantizar el adecuado control de los flujos de dinero del inversionista y el promotor - transferencias de dinero, pago de rentabilidad, etc.-, se tiene que los administradores de las plataformas han optado por realizarlo a través de una cuenta bancaria - por ejemplo, una cuenta escrow - o consti- tuir un fideicomiso de administración con una entidad financiera.

Como se puede inferir, se tendrá como relación jurídica principal la producida entre el promotor y los inversionistas que tendrá la naturaleza de un contrato de mutuo o préstamo dinerario, en virtud del cual este último otorga una suma dineraria a favor del promotor a cambio de obtener una rentabilidad que se encuentra materializada en el pago de intereses.

De otro lado, las relaciones jurídicas producidas entre el administrador de la plataforma con el promotor y los inversionistas, las mismas que se canalizan mediante contratos de mandato ${ }^{23}$ con representación ${ }^{24}$, son las siguientes: (a) mediante un contrato de mandato suscrito entre el administrador de la plataforma y los inversionistas, este último autoriza a aquella a que con sus fondos, a su nombre, cuenta y riesgo lleve a cabo operaciones de préstamo; $y$, (b) mediante un contrato de mandato suscrito entre el administrador de la plataforma y el promotor - receptor de la inversión-, este último autoriza a aquella a solicitar financia-

17. “Difondy", Difondy, acceso el 21 de marzo de 2021, https://www.difondy.com/

18. "Plataforma de inversión | AirOrHouse", AirOrHouse, acceso el 21 de marzo de 2021, https://airorhouse.com/

19. "Prestamype | Financiamiento accesible y justo para mypes", Prestamype, acceso el 21 de marzo de 2021, https:// www.prestamype.com/

20. "Préstamos agrícolas digitales", Agroinvesting, acceso el 21 de marzo de 2021, https://www.agroinvesting.lat/

21. "Finsmart - Factoring hecho simple - 100\% peruano", Finsmart, acceso el 21 de marzo de 2021, https://finsmart. pe/

22. "Creafunding|F6S", Creafunding, acceso el 21 de marzo de 2021, https://www.f6s.com/creafunding

23. En el artículo 1790 del Código Civil se define al contrato de mandato como una modalidad del contrato de prestación de servicios nominados, en tanto el mandatario asume la obligación de realizar uno o más actos jurídicos, por cuenta y en interés del mandante. Tratándose del caso específico del contrato de mandato con representación, se tiene que el mandatario actúa en nombre del mandante para representarlo en todo aquello que le sea atribuido por el mandatario; de tal forma que los derechos y obligaciones derivados actos recaen directamente en el mandante.

24. Excepcionalmente se han evidenciado algunos casos en los cuales se suscribe un mandato sin representación; en cuyo caso será de aplicación lo previsto en el artículo 1809 del Código Civil, en virtud del cual el mandatario actuará en nombre propio para realizar los actos que hayan sido determinados por el mandante, adquiriendo derechos y asumiendo obligaciones derivadas del mandato. 
mientos dinerarios a su nombre y por su cuenta y riesgo.

Para tal efecto, el administrador de la plataforma cobrará comisiones como contraprestación por las actividades llevadas a cabo en nombre y por cuenta y riesgo del operador y los inversionistas - por ejemplo, comisión por traslado de fondos, comisión de adhesión, comisión de mantenimiento, comisión por gestión de cobranza, comisión por intercambio de portafolios, entre otros-.

Atendiendo a las consideraciones de las secciones II y III se puede esquematizar la estructura del negocio de crowdfunding — bajo la cual se estaría operando en el mercado peruano- de la siguiente manera:

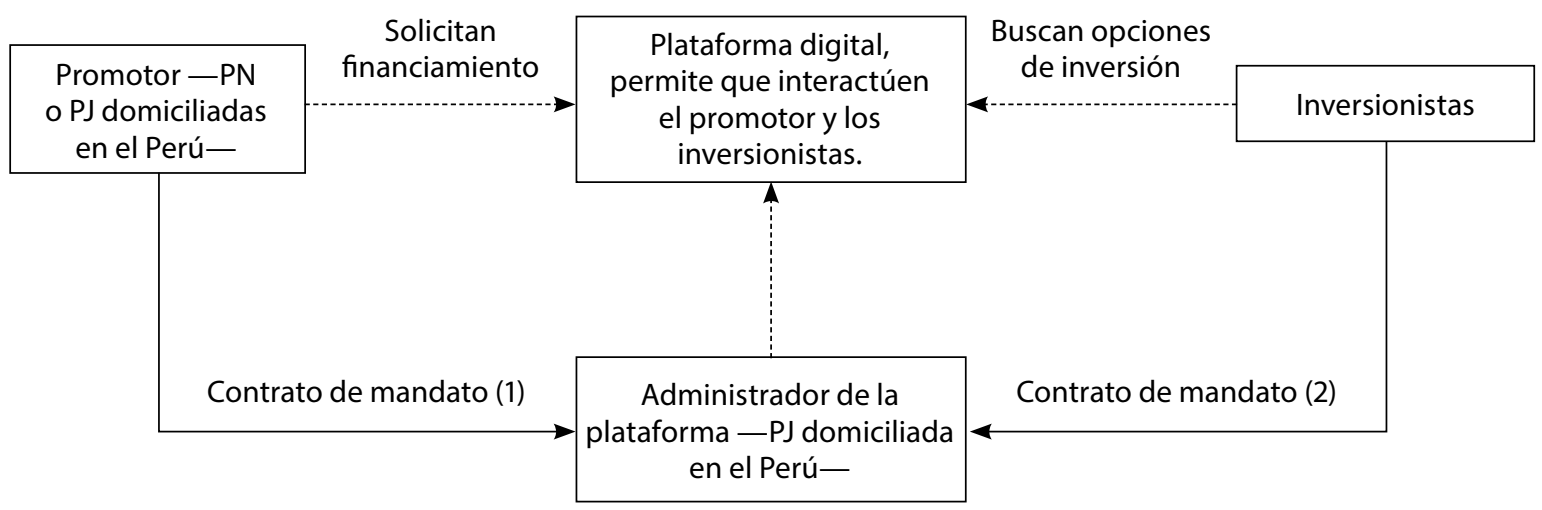

\section{PRINCIPALES ASPECTOS TRIBUTARIOS A TENER EN CONSIDERACIÓN}

Como se señaló anteriormente, el impacto tributario asociado a una operación de crowdfunding variará dependiendo de los sujetos que participen y el tipo de relaciones jurídicas existentes dentro de su estructura. Tomando en consideración las afirmaciones anteriores, nos parece conveniente realizar este análisis en primer lugar tomando en consideración la relación jurídica existente entre el promotor y los inversionistas $y$; en segundo lugar, las relaciones jurídicas que se llevarían a cabo con el administrador de la plataforma.

\subsection{Respecto a la relación jurídica realizada entre el promotor y los inversionistas.}

Como primer punto se deberá determinar la naturaleza de la operación. Así, se tiene que el inversionista transferirá dinero a favor del promotor para que a su vez éste lo utilice como capital para el desarrollo de su proyecto, la constitución de una nueva empresa o haga crecer su negocio ya existente. Ello calificará como un contrato de mutuo de acuerdo con lo previsto en los artículos 1648 y 1663 del Código Civil.

Por lo tanto, nos encontraremos frente a un préstamo de dinero en virtud del cual la rentabilidad obtenida por los inversionistas estará dada por el pago adicional que realice el promotor por el uso del dinero en el tiempo, el mismo que calificará como un interés ${ }^{25}$ para efectos del Impuesto a la Renta - IR.

Desde la perspectiva tributaria, se deberá tener en consideración la característica del promotor y de los inversionistas. Tratándose del primero,

25. De acuerdo con lo previsto en el artículo 1242 del Código Civil, el interés compensatorio constituye la contraprestación por el uso del dinero o cualquier otro bien. Dicha definición ha sido recogida por la Administración Tributaria en los Informes No 36-2017-SUNAT/34000, 103-2007-SUNAT/2B0000, 140-2009-SUNAT/2B0000, 099-2015-SUNAT/5D0000 y 32-2007-SUNAT/2B0000. 
según lo revisado en las secciones II y III, se trataría de una persona jurídica domiciliada en el Perú. Por su parte, los inversionistas podrían ser personas naturales o jurídicas domiciliadas o no domiciliadas en territorio peruano - eventualmente podría presentarse el caso que sea un ente jurídico- ${ }^{26}$

Tratándose de personas naturales domiciliadas, dichos ingresos calificarán como rentas de segunda categoría gravadas con la tasa efectiva de $5 \%$, las mismas que deberán ser imputadas al ejercicio gravable en el cual se perciban. Por su parte, tratándose de personas jurídicas constituidas en el país los intereses que cobren constituirán rentas de tercera categoría gravadas con la tasa de $29.5 \%$ - que se imputarán cuando se devenguen - siempre que se trate de una empresa sujeta al Régimen General del IR; $y$ en caso se trate de una empresa sujeta el Régimen MYPE —-mediana y pequeña empresa- Tributario, las primeras 15 UIT $^{27}$ se encontraran gravadas con la tasa de $10 \% .^{28}$

De otro lado, tratándose de sujetos no domiciliados, los intereses ${ }^{29}$ que sean pagados se encontrarán gravados vía retención aplicando la tasa de 4.99\%; ya sea que se trate de una persona natural ${ }^{30}$ o una persona jurídica ${ }^{31}$ — siempre que se cumplan con los requisitos expresamente previstos en la Ley del IR y su Reglamento-. En el caso en particular donde coincidan como promotor e inversionistas personas naturales la tasa aplicable será de 5\%.32,33

26. Entiéndase como entes a los fondos de inversión, fondos mutuos de inversión en valores, así como fideicomisos bancarios y de titulización.

27. Nota del Editor: Unidad Impositiva Tributaria, que para el ejercicio 2021 asciende a s/ 4,400.

28. Ello de acuerdo con lo previsto en el Decreto Legislativo 1269 que crea el Régimen MYPE Tributario del IR.

29. De acuerdo con lo previsto en el inciso c) del artículo 9 de la Ley del IR, se consideran como rentas de fuente peruana las producidas por capitales, así como los intereses, comisiones, primas y toda suma adicional al interés pactado por préstamos, créditos u otra operación financiera, cuando el capital esté colocado o sea utilizado económicamente en el país; o cuando el pagador sea un sujeto domiciliado en el país.

30. El inciso d) del artículo 54 de la Ley del IR ha previsto que la tasa de $4.99 \%$ es aplicable a los intereses cuando los pague o acredite un generador de rentas de tercera categoría que se encuentre domiciliado en el país. Dicha tasa será aplicable siempre que entre las partes no exista vinculación o cuando los intereses no deriven de operaciones realizadas desde o a través de países o territorios de baja o nula imposición, en cuyo caso se aplicará la tasa de $30 \%$.

31. El inciso e) del artículo 56 de la Ley del IR establece que los requisitos para aplicar la tasa de $4.99 \%$ se deberá observar entre otros los siguientes requisitos: (a) los créditos se encuentren destinados a cualquier finalidad relacionada con el giro del negocio o actividad gravada; (b) en caso de préstamo en efectivo, que se acredite el ingreso de la moneda extranjera al país; (c) que el crédito no devengue un interés anual a rebatir a la tasa preferencial predominante en la plaza de donde provenga más tres puntos, y; (d) no aplica para los intereses que abonen al exterior las empresas privadas del país por créditos concedidos por una empresa del exterior con la cual se encuentren vinculadas, o de los intereses que abonen al exterior las empresas privadas del país por créditos concedidos por un acreedor cuya intervención tiene como propósito encubrir una operación de crédito entre partes vinculadas.

32. El inciso e) del artículo 54 de la Ley del IR señala que, tratándose de otras rentas provenientes de capital, la tasa aplicable será de $5 \%$.

33. El tratamiento tributario aplicable a los inversionistas que tengan la calidad de sujetos no domiciliados para efectos de la Ley del IR podría variar sustancialmente en caso sean residentes de países con los cuales el Perú haya suscrito Convenios Para Evitar la Doble Imposición. Actualmente - a la fecha de edición de este artículo, 21 de marzo de 2021—, el Perú ha suscrito Convenios con los siguientes países: Chile, Canadá, Brasil, México, Corea, Suiza, Portugal, y es parte de la Comunidad Andina conformada por Bolivia, Colombia, y Ecuador. 
Por otro lado, tratándose del promotor y siempre que se trate de una persona jurídica domiciliada en el Perú, los intereses pagados podrán ser deducidos para efectos de la determinación de su renta neta. ${ }^{34,35}$ Ello también se aplicará aún en el supuesto que se encuentre acogida al Régimen MYPE Tributario. Nótese que, de acuerdo con lo previsto en el Decreto de Urgencia 013-2020, los promotores deberán ser sujetos domiciliados en el país - personas naturales o jurídicas-.

En cuanto al Impuesto General a las Ventas IGV, la operación de financiamiento se encontrará gravada con dicho impuesto aplicando la tasa de $18 \%$ siempre que se presenten las siguientes situaciones: (a) el inversionista se encuentre domiciliado en el país y sea perceptor de rentas de tercera categoría, en cuyo caso tendrá la calidad de contribuyente o; (b) el inversionista sea una persona no domiciliada que desarrolla actividad empresarial, y el promotor sea una persona jurídica domiciliada —en caso sea una persona natural domiciliada deberá cumplir con el criterio de habitualidad previsto en la Ley del IGV y su Reglamento-, en estos casos la calidad de contribuyente la tendrá el promotor.

\subsection{Respecto a las relaciones jurídicas reali- zadas entre el administrador de la plata- forma, el promotor y los inversionistas.}

Generalmente, el administrador de la plataforma digital opta por suscribir contratos de mandato con representación en virtud de los cuales en su calidad de mandatario se compromete a realizar diferentes operaciones a nombre y por cuenta y riesgo del promotor y de los inversionistas, a efectos de llevar a cabo las operaciones de préstamo.

Al respecto, el artículo 1790 del Código Civil señala que, por el mandato, el mandatario se obliga a realizar uno o más actos jurídicos por cuenta y en interés del mandante. Respecto al caso específico del mandato con representación, en su artículo 1806 precisa que le serán aplicables las normas correspondientes a la representación y que el mandatario debe actuar en nombre del mandante.

Conforme a ello, en un contrato de mandato con representación resultará de aplicación lo previsto en el artículo 160 del Código Civil, el cual prevé que el acto jurídico celebrado por el mandatario dentro de los límites de las facultades que se hayan conferido produce efectos directamente respecto del mandante.

Por lo tanto, las implicancias fiscales que se desprendan de los actos jurídicos realizados por el administrador de la plataforma en su calidad de mandatario producirán sus efectos directamente sobre el promotor y los inversionistas mandantes-.

En el caso en concreto, se tiene que como contraprestación por las diferentes actividades llevadas a cabo - por ejemplo, gestión de cobranza, promoción de oportunidades de inversión, etc.- a favor del promotor y de los in-

34. Tomando en consideración que generalmente serán promotores empresas en etapas iniciales, se deberá tener en consideración lo previsto en el inciso g) del artículo 37 de la Ley del IR, el cual establece que serán deducibles para efectos de la determinación de la renta los gastos de organización, los gastos preoperativos iniciales, los gastos preoperativos originados por la expansión de las actividades de la empresa; los intereses devengados durante el periodo preoperativo, a opción del contribuyente, podrán deducirse en el primer ejercicio o amortizarse proporcionalmente en el plazo máximo de diez años.

35. No resultarán deducibles los intereses netos provenientes de endeudamientos — sean estos efectuados entre partes independientes o vinculadas - que excedan el 30\% del EBITDA —Earnings Before Interest, Taxes, Depreciation, and Amortization - del ejercicio anterior. A tal efecto, se entenderá por interés neto el monto de los gastos por intereses neto del monto de los ingresos por intereses, computables para determinar la renta neta; y por EBITDA la renta neta luego de efectuada la compensación de pérdidas más los intereses netos, depreciación y amortización. 
versionistas, el administrador de la plataforma cobraría una comisión, la que calificaría como un ingreso por servicios que se encontrará gravado con el IR con la tasa de $29.5 \%$ siempre que se trate de una empresa sujeta al Régimen General del IR. En el supuesto que se trate de una compañía dentro del Régimen MYPE Tributario, el impuesto a pagar se determinará aplicando las tasas de manera progresiva; esto es, primero se aplicará la tasa de $10 \%$ hasta las primeras 15 UIT y superado dicho importe se aplicará la tasa de $29.5 \%$.

De otro lado, tratándose del IGV, toda vez que nos encontraríamos frente a la prestación de un servicio, las comisiones cobradas se encontrarán gravadas con dicho impuesto con la tasa de $18 \%$, siendo el contribuyente el administrador de la plataforma.

Finalmente, se deberá tener en especial consideración lo previsto en el artículo 16 del Código Tributario, el cual dispone que los mandatarios se encontrarán obligados a pagar los tributos y cumplir con las obligaciones formales en calidad de representantes, con los recursos que administren o dispongan.

En ese sentido, los mandatarios en su calidad de representantes estarán obligados al cumplimiento de obligaciones y deberes tributarios, en la medida en que tienen responsabilidad de manejo y disposición de los recursos del mandante. Dicho criterio ha sido compartido por la Administración Tributaria en el Informe No 103-2016-SUNAT, en el cual concluyó que los mandatarios deberán cumplir con retener el IR que grave aquellas rentas correspondientes a sus mandantes, en aquellos casos en los cuales califiquen como rentas de segunda categoría. ${ }^{36}$

\subsection{Respecto a la obligación de emitir com- probantes de pago.}

De acuerdo con el Reglamento de Comprobantes de Pago - RCP éstos se emitirán cada vez que se lleve a cabo, entre otros, una prestación de servicios como es el caso de un financiamiento o la prestación de servicios por parte del administrador de la plataforma tratándose de las operaciones de crowdfunding.

Se deberá tener en consideración que, en operaciones de préstamo de dinero, quien realiza la emisión del comprobante de pago es quien efectúa el financiamiento; por lo tanto, en el caso en concreto serían los inversionistas quienes deberán emitir los comprobantes de pago a favor del promotor.

En caso el promotor sea una persona jurídica domiciliada, contar con el comprobante de pago resulta de especial importancia, ya que el mismo le permitirá deducir como gasto los intereses pagados a los inversionistas. En el supuesto que se trate de una persona jurídica domiciliada deberá emitir una factura, y tratándose de una persona natural domiciliada que no realice dichas operaciones de forma habitual, deberá emitir el Formulario № 820 - Comprobante por operaciones no habituales.

El cumplimiento de la obligación formal de emisión de comprobantes de pago tiene especial complejidad en el caso de los negocios de crowdfunding debido la pluralidad de inversionistas, y más cuando algunos sean personas naturales o jurídicas no domiciliadas. A efectos de mitigar ello, eventualmente el administrador de la plataforma podría ser quien emita los comprobantes de pago a favor del promotor, a

36. Adicionalmente, el Código Tributario imputa responsabilidad solidaria al mandatario cuando por dolo, negligencia grave o abuso de facultades se dejen de pagar las deudas tributarias. Cabe tener presente que la Administración Tributaria, mediante el Informe $N^{\circ} 339-2003-S U N A T$, ha establecido que la responsabilidad solidaria en los supuestos a que se refieren los numerales 2, 3 y 4 del artículo 16 del Código Tributario — dentro del cual se encuentra la responsabilidad solidaria del mandatario — solo podrá alcanzar al pago de las obligaciones tributarias a las que estaban obligados a cumplir dichos sujetos en su calidad de representantes; esto es, aquellas cuyo incumplimiento se haya verificado durante su gestión, independientemente de la fecha de emisión y notificación del acto mediante el cual se exija su cumplimiento. 
nombre y por cuenta de los inversionistas en virtud de los contratos de mandato con representación suscritos.

Sin perjuicio de ello, se deberá tener en consideración que los inversionistas no domiciliados deberán emitir comprobantes de pago observando los siguientes requisitos: (a) nombre, denominación o razón social; (b) domicilio, (c) naturaleza u objeto de la operación; (d) fecha; $y$, (d) monto de la operación. ${ }^{37}$

Por último, tratándose de los servicios prestados por parte del administrador de la plataforma a favor del promotor y de los inversionistas, este deberá emitir los comprobantes de pago correspondientes por las comisiones cobradas en calidad de contraprestación.

\subsection{Consideraciones adicionales.}

Ahora bien, de nuestra revisión de un grupo de plataformas digitales de crowdfunding que operan en el mercado peruano se observa que en algunos casos se ofrece acceder a financiamientos participando en operaciones de factoring, esto es mediante la cesión de documentos de crédito - facturas negociables ${ }^{38}$ - a múltiples inversionistas.
Si bien el propósito de este artículo no es ahondar en el tratamiento tributario aplicable a las operaciones de factoring que eventualmente se lleven a cabo, consideramos pertinente señalar las principales implicancias fiscales que se originarían para cada uno de los partícipes.

Para efectos del IR, la naturaleza de la rentabilidad obtenida por los inversionistas dependerá si al momento de adquirir los documentos de crédito asume el riesgo del deudor cedido o no. En el primer caso, la diferencia entre el valor nominal del crédito y el valor de transferencia constituirá un ingreso por servicios, caso contrario dicha diferencia será considerando como un ingreso por intereses.

Respecto a la tasa aplicable, se deberá tener en consideración que si el inversionista es una persona natural domiciliada en el Perú el ingreso obtenido estará gravado con la tasa definitiva de $5 \% .{ }^{39}$ Por su parte, en caso el inversionista sea una persona jurídica domiciliada en el país la rentabilidad obtenida como interés o como contraprestación por un servicio constituirá una renta empresarial gravada con la tasa de $29.5 \%$.

En la misma línea, en el caso de sujetos no domiciliados, bajo la calificación de la cesión de

37. El artículo 51-A de la Ley del IR ha establecido que, como mínimo, dichos requisitos deberán encontrase en los comprobantes emitidos en el exterior a efectos de que permitan acreditar los gastos incurridos en el extranjero.

38. De acuerdo con la Ley 29623 - Ley que Promueve el Financiamiento a través de la Factura Comercial—, la Factura Negociable es un título valor a la orden transferible por endoso o un valor representado y transferible mediante anotación en cuenta en una Institución de Compensación y Liquidación de Valores — ICLV_ como CAVALI. Dichas facturas negociables se originan en la compraventa $u$ otras modalidades contractuales de transferencia de propiedad de bienes o en la prestación de servicios e incorporan el derecho de crédito respecto del saldo del precio o contraprestación pactadas por las partes.

39. De acuerdo con lo previsto en el artículo 5 de la Ley 30532 - Ley que promueve el desarrollo de mercado de capitales, modificada por el Decreto Legislativo 1371 publicado el 2 de agosto de 2018 y vigente desde el 01 de enero de 2019-, los ingresos por servicios obtenidos por el factor o adquirente de una factura negociable está sujeto a una tasa de retención definitiva del IR de 5\% siempre que sea una persona natural, sucesión indivisa o sociedad conyugal que optó por tributar como tal, domiciliada en el país o una empresa unipersonal constituida en el exterior. Agrega que la misma tasa será aplicable cuando la operación es realizada a través de un fondo de inversión, fideicomiso bancario y de titulización, siempre que dichos ingresos sean atribuidos a una persona natural, sucesión indivisa o sociedad conyugal que optó por tributar como tal, domiciliada en el país, o una empresa unipersonal constituida en el exterior. 
créditos sin recurso como un servicio el gravamen aplicable será de 30\%; y tratándose de una cesión de créditos con recurso la tasa aplicable sería de $4.99 \% .^{40}$

Tratándose del IGV, únicamente se encontrarán gravadas las operaciones de factoring en las cuales el adquirente sea una persona jurídica y no asuma el riesgo crediticio del deudor en tanto se entenderá que nos encontramos frente a un servicio. Nótese que, en este caso en particular, se entenderá que el servicio se configura a partir del momento en que se produzca la devolución del crédito al transferente o este recompre el mismo. Caso contrario, no se configuraría y la operación no se encontraría gravada. ${ }^{41}$

Por su parte, respecto a las operaciones llevadas a cabo entre el administrador de la plataforma con el promotor y los inversionistas, se tiene que la operatividad es similar a la llevada a cabo cuando se trata de un financiamiento mediante un préstamo de dinero; esto es, se suscriben contratos de mandato a efectos de que el administrador de la plataforma pueda llevar a cabo todas las acciones necesarias para realizar las operaciones de factoring, cobrando para dichos efectos una comisión.

\section{CONCLUSIONES}

La utilización de las TIC en el mercado financiero ha cobrado notoriedad en Perú, toda vez que vienen siendo incorporadas tanto por las Empresas del Sistema Financiero - por ejemplo: empresas bancarias, empresas financieras, cajas municipales de ahorro y crédito, etc.-, así como por otras empresas que mediante nuevos modelos de negocio ofrecen servicios de financiamiento, como son las plataformas digitales de crowdfunding.
El modelo de negocio de crowdfunding permite acceder a financiamientos mediante el cual las personas o empresas - sobre todo orientado a los nuevos emprendimientos o startupspuedan acceder a fondos provistos por una pluralidad de pequeños inversionistas; y que además se caracteriza por que la operación se lleva a cabo esencialmente a través de una plataforma digital.

Tomando en consideración que nos encontramos frente a un modelo de negocio relativamente nuevo, resulta esencial tener claro las implicancias tributarias para las empresas que han visto una alternativa atractiva a donde recurrir para obtener un financiamiento, así como para los inversionistas que buscan obtener algún tipo de rentabilidad y desean tener seguridad respecto al retorno de su inversión.

Toda vez que no existe una modalidad típica bajo la cual se lleve a cabo los negocios de crowdfunding, las implicancias fiscales estarán dadas por las relaciones jurídicas que se lleven a cabo y el tipo de sujetos que participen en ellas. De nuestra revisión se han evidenciado que en general se presentan las siguientes: (a) contrato de préstamo o mutuo dinerario entre el promotor y los inversionistas, y (b) prestación de servicios por parte del administrador de la plataforma a favor del promotor y los inversionistas.

Es pertinente evaluar algunas propuestas de incentivos fiscales con el objetivo de promover la participación de inversionistas en operaciones de crowdfunding: en otras legislaciones se están evaluando opciones para incentivar la inversión en nuevas empresas otorgando ciertas ventajas fiscales como deducciones adicionales o una imposición limitada — tasa reducida — sobre la rentabilidad obtenida por los inversionistas.

40. La tasa de $4.99 \%$ se encontrará condicionada al cumplimiento de los requisitos expresamente recogidos en el artículo 54 y 56 de la Ley del IR, caso contrario la tasa de retención aplicable sería de $30 \%$.

41. En ese sentido se ha pronunciado la Administración Tributaria mediante el Informe $N^{\circ} 124-2020-S U N A T / 7 T 0000$ concluyendo que la devolución del crédito o su recompra dan forma, en este caso, a la operación gravada; constituyendo, por tanto, parte del aspecto material del hecho imponible, de modo tal que si no acontece la devolución o recompra de crédito no habrá operación gravada. 